DRAFT VERSION DECEMBER 5, 2018

Preprint typeset using LATEX style emulateapj v. 11/12/01

\title{
THE UNIVERSAL GAS MASS FRACTION IN CLUSTERS OF GALAXIES
}

\author{
LAUREnCE P. DAVid, Christine Jones \& William Forman \\ Harvard-Smithsonian Center for Astrophysics, 60 Garden St., Cambridge, MA 02138 \\ ldavid@head.cfa.harvard.edu \\ Draft version December 5, 2018
}

\begin{abstract}
We obtained a deep $150 \mathrm{ksec}$ Chandra observation of the optically selected cluster of galaxies, RCS 2318+0034, to investigate the gas mass fraction in this system. Combining our deep Chandra observation with an archival $50 \mathrm{ksec}$ observation, we derive gas mass fractions of $f_{\text {gas }}=0.06 \pm .02$ and $0.10 \pm .02$ within $r_{2500}$ and $r_{500}$, respectively. The gas mass fraction in RCS $2318+0034$ within $r_{500}$ is typical of X-ray selected clusters. Further study shows that the large scale properties of RCS 2318+0034, including the relations between gas mass, Xray luminosity and gas temperature are also consistent with the observed correlations of X-ray selected clusters. However, the gas mass fraction within $r_{2500}$ is less than most X-ray selected clusters, as previously reported. The deep Chandra image of RCS $2318+0034$ shows that this system is presently undergoing a major merger which may have an impact on the inferred gas mass fraction within $r_{2500}$.
\end{abstract}

Subject headings: galaxies:clusters:general - intergalactic medium - X-rays:galaxies:clusters cosmology:observations

\section{INTRODUCTION}

The current matter in rich clusters of galaxies was accumulated from regions that would presently span approximately $10 \mathrm{Mpc}$ and should comprise a fair sample of the Universe. It has been known for some time that the dominant component of baryonic matter in rich clusters is the hot X-ray emitting gas (David et al. 1990; White \& Fabian 1995; David et al. 1995; Jones \& Forman 1999; Ettori et al. 2003; Allen et al. 2004; Vikhlinin et al. 2006; Allen et al. 2008; Sun et al. 2009; Vikhlinin et al. 2009). White et al. (1993) showed that the observed high gas mass fraction in clusters has significant cosmological consequences regarding the total mass density of the universe. The segregation of baryons between stars and hot gas depends on both the radius within a group or cluster and the total mass of the system. The ratio of gas mass to stellar mass increases from approximately unity in the cores of groups up to more than than a factor of five at large radii in the richest and hottest clusters (David et al. 1995, Gonzales et al. 2007). In addition, since the gas is more extended than the dark matter, the gas mass fraction increases with radius within groups and clusters (David et al. 1995, White \& Fabian 1995, Allen et al. 2004, Vikhlinin et al. 2006). The greater extent of the gas, relative to the stellar and dark matter, probably arises from some form of non-gravitational heating (e.g., mechanical AGN heating and supernova driven galactic winds) which breaks the selfsimilarity between groups and clusters (Ponman et al. 1999, Arnaud \& Evrard 1999, Lloyd-Davies et al. 2000, Bialek et al. 2001, Neumann \& Arnaud 2001, Finoguenov et al. 2002, Voit et al. 2005, Kravtsov et al. 2005, Davé et al. 2008, Bode et al. 2009).

Most studies of nearby clusters find that the gas mass fraction approaches a nearly constant value of $f_{\text {gas }} \approx 13 \%$ at large radii in the hottest and most massive clusters (David et al. 1995, White \& Fabian 1995, Allen et al. 2004, Vikhlinin et al. 2006, Sun et al. 2009). This is slightly less than the WMAP value of $17 \%$. Based on these findings, there have been several cosmological studies whose results are based on the assumption that the gas mass fraction in rich clusters is independent of redshift (Ettori et al. 2003, Allen et al. 2004, Ferramacho \& Blanchard
2007, Ettori et al. 2009, Juett et al. 2010). All of these studies have found results consistent with the concordant $\Lambda \mathrm{CDM}$ cosmological model. However, a recent analysis of Chandra observations of 13 optically selected, moderate redshift clusters culled from the Red-Sequence Cluster Survey (RCS; Gladders $\&$ Yee 2005) by Hicks et al. (2008), reports a significant scatter in the gas mass fraction of clusters with average gas mass fractions of $f_{\text {gas }}=4.8 \%$ at $r_{2500}$ and $6.8 \%$ at $r_{500}$ (where $r_{\Delta}$ is the radius within which the average mass density of the cluster is $\Delta$ times the critical density of the universe at the redshift of the cluster). If this result is more representative of clusters compared to previous studies of X-ray selected clusters, then it would make precision cosmological studies based on the assumption of a universal gas mass fraction in clusters problematic. The cluster with the lowest gas mass fraction in the Hicks et al. (2008) sample is RCS $2318+0034$ with reported values of $f_{\text {gas }}=4.0 \%$ and $1.5 \%$ at $r_{2500}$ and $r_{500}$, respectively. Due to the cosmological consequences of this result, we obtained an additional $150 \mathrm{ksec}$ Chandra observation of RCS 2318+0034 in AO-11 to better constrain the gas mass fraction in this system.

This paper is organized as follows. Section 2 contain a detailed discussion about the Chandra data analysis. Section 3 presents evidence for a recent merger in RCS 2318+0034 and $\S 4$ examines the X-ray surface brightness distribution. All spectral analysis of the Chandra data is presented in $\$ 5$. Section 6 gives the gas mass, total gravitating mass and gas mass fraction at $r_{2500}$ and $r_{500}$ and a comparison of our work with previous estimates is given in $\$ 7$. Our main results and their cosmological implications are summarized in $\S 8$.

\section{DATA ANALYSIS}

RCS 2318+0034 was observed with the Chandra ACIS-S detector on May 3, 2005 (ObsID 4938) and with the ACISI detector on Aug. 7, 2009 (ObsID 11718). Both observations were taken in very faint (VF) telemetry mode. All data were re-processed with CIAO 4.3 and CALDB 4.4.6. We used acis_process_events to apply the latest CTI and gain corrections and filtered the VF mode data to reduce the charged particle background. During the ACIS-S observation, the I2, I3, S1, S2 and $\mathrm{S} 3$ chips were turned on and the cluster was positioned on 

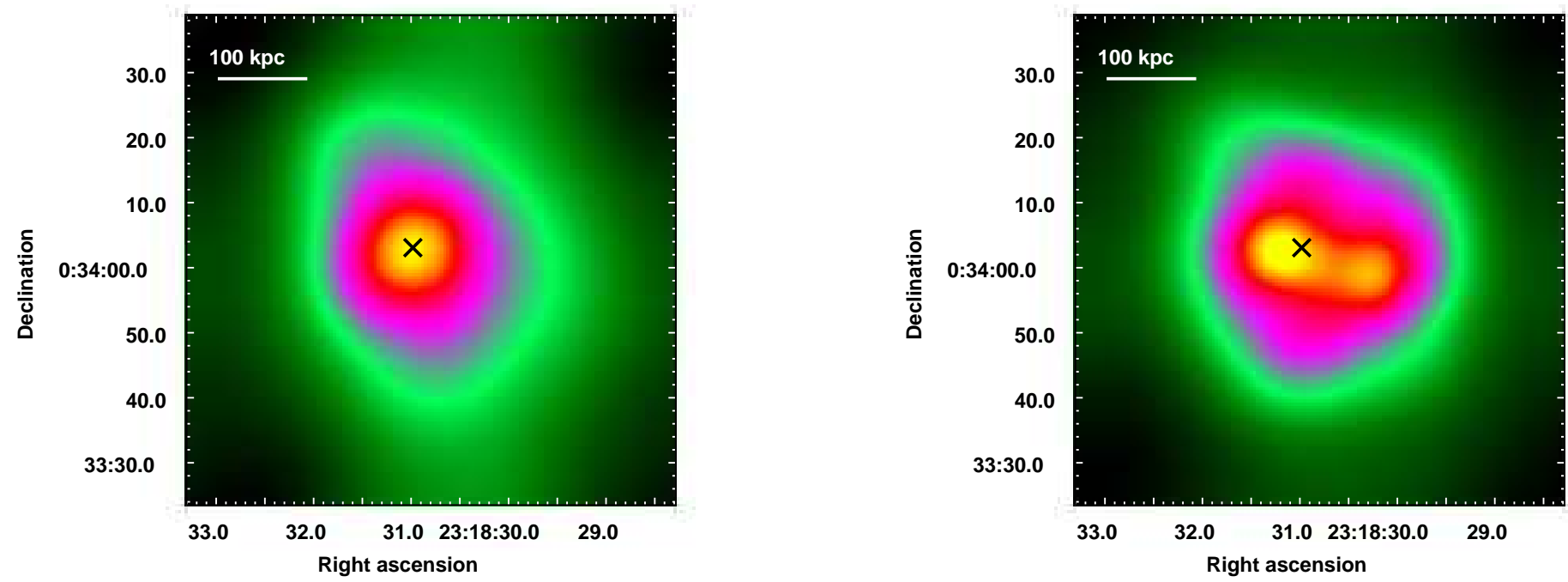

FIG. 1.- left: hard band (2.0-6.0 kev) adaptively smoothed image, and right: soft band (0.5-2.0 keV) adaptively smoothed image of RCS $2318+0034$. The cross marks the location of the peak surface brightness of the main cluster in the hard band image.

the S3 chip. During the ACIS-I observation, the I0, I1, I2, I3 and $\mathrm{S} 3$ chips were turned on and the cluster was positioned on the $\mathrm{I} 3$ chip. Light curves were generated in the $2.5-7.0 \mathrm{keV}$ and 9.0-12.0 keV energy bands for the BI chip S1 during the ACISS observation and for the BI chip S3 during the ACIS-I observation. All time intervals with background rates in these energy bands exceeding $20 \%$ of the quiescent background rate were excised from further analysis. The resulting cleaned exposure times for the ACIS-S and ACIS-I observations are 50,155sec and $143,275 \mathrm{sec}$. The appropriate ACIS blank field background files (Epoch D for the ACIS-S observation and Epoch E for the ACIS-I observation) were extracted from the CALDB and reprojected onto the sky to match the two RCS $2318+0034$ observations. The same VF background screening was applied to the background data sets by only including events with "status $=0$ ". Finally, the exposure times for each chip in the background data sets were adjusted to produce the same $9-12 \mathrm{keV}$ count rates as in the two RCS $2318+0034$ observations.

Molecular contamination has been building up on the cold ACIS filters since launch. This only affects the ACIS QE below $2 \mathrm{keV}$. Since the ACIS-I observation of RCS 2318+0034 was taken more recently than the observations used to compile the background files, the throughput of ACIS at low energies in the RCS $2318+0034$ observation is less than it was during the background observations. To correct for this affect, we extracted spectra from a source free region on I3 beyond the virial radius of the cluster in the ACIS-I observation of RCS $2318+0034$ and the I3 blank field background file. We found that the count rate in the $0.3-6.0 \mathrm{keV}$ energy band (i.e., the energy band we use to generate surface brightness profiles) during the RCS $2318+0034$ observation is still $4.6 \%$ less than the count rate in the I3 blank field data set even after adjusting the exposure time in the background data set to produce the same 9-12 keV count rate. We therefore reduced the count rate in the I3 background data set by $4.6 \%$ before subtracting the background. We repeated this procedure for all 5 chips (all 4 ACISI chips from the ACIS-I observation and the S3 chip from the ACIS-S observation) used to generate background-subtracted surface brightness profiles for RCS $2318+0034$. For all spectral analysis, we used the CIAO task specextract which extracts source and background spectra and computes photon-weighted effective area and response files.

Due to the lack of measured galaxy redshifts for RCS $2318+0034$, we adopt a redshift of $\mathrm{z}=0.78$ (which is the same as that used in Hicks et al. 2008) derived from the energy centroid of the $\mathrm{H}$-like and He-like $\mathrm{Fe}-\mathrm{K} \alpha$ emission lines in the ACIS spectrum. Assuming the standard $\Lambda \mathrm{CDM}$ cosmology with $\mathrm{H}_{0}=70 \mathrm{~km} \mathrm{~s}^{-1} \mathrm{Mpc}^{-1}, \Omega_{M}=0.3$ and $\Omega_{\Lambda}=0.7$, gives a luminosity distance of $D_{L}=4,870 \mathrm{Mpc}$ to RCS $2318+0034$ and $1^{\prime \prime}=7.45 \mathrm{kpc}$ in the rest frame of the cluster.

\section{X-RAY MORPHOLOGY}

Adaptively smoothed ACIS images of the co-added RCS $2318+0034$ observations in a hard $(2.0-6.0 \mathrm{keV})$ and soft $(0.5-$ $2.0 \mathrm{keV}$ ) energy band are shown in Figure 1. In the hard band image, RCS $2318+0034$ appears to be a fairly relaxed hot cluster, while the soft band image reveals that RCS $2318+0034$ is undergoing a merger with a smaller and cooler system that is presently located about $100 \mathrm{kpc}$ to the west of the dominant cluster. The " $\mathrm{x}$ " in both figures indicates the location of the peak in the hard band surface brightness of the main cluster. The soft energy band image shows that the cool gas in the center of the main cluster has been displaced toward the east during the merger, possibly due to merger-induced sloshing (Ascasibar \& Markevitch 2006).

Since there are insufficient counts in the ACIS data to generate a temperature map, we generated a hardness ratio image by adaptively smoothing the raw $0.5-2.0 \mathrm{keV}$ and $2.0-6.0 \mathrm{keV}$ images with the same kernel and then divided the two images. The resulting hardness ratio image is shown in Figure 2 along with surface brightness contours derived from the soft band image shown in Figure 1. The hardness ratio image shows that there is an elongated region of softer X-ray emission associated with the merging cooler system. The coolest emission from the main cluster is slightly displaced toward the east from the cluster core. The hardest $\mathrm{X}$-ray emission arises from the region between the two merging cores. The spectral analysis presented below shows that this hard X-ray emission probably originates from a merger induced shock. 


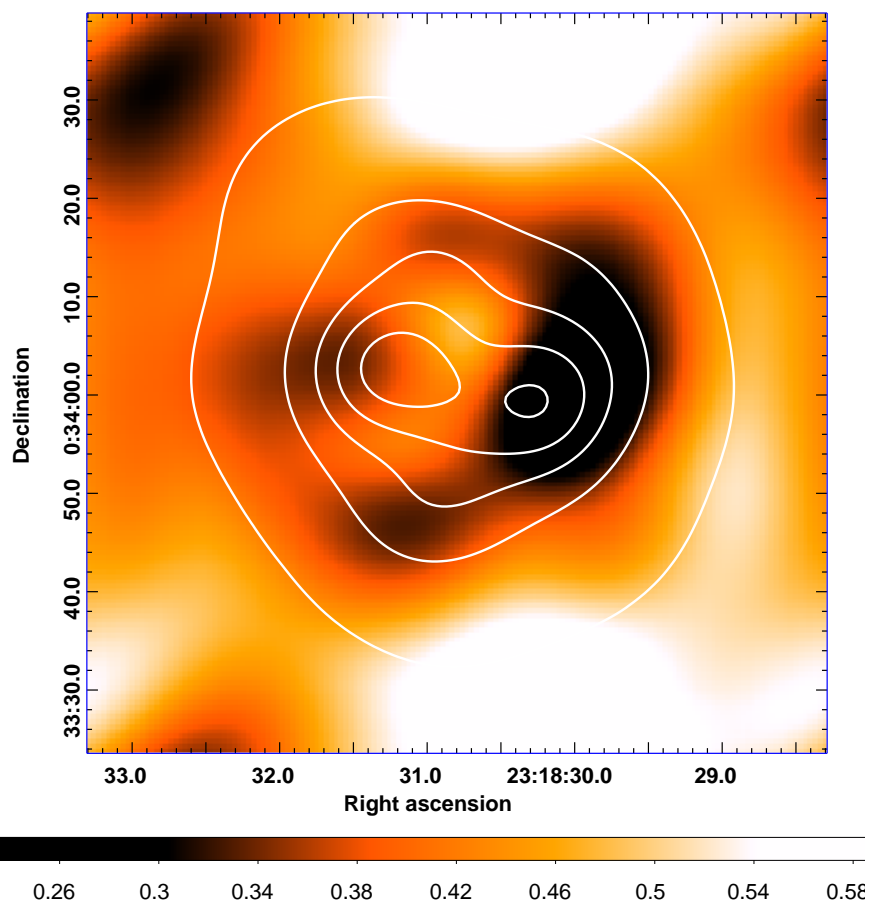

FIG. 2.- Hardness ratio image along with surface brightness contours derived from the soft band image shown in Fig. 1.

\section{SURFACE BRIGHTNESS PROFILE}

Due to the on-going merger in RCS 2318+0034, we extracted surface brightness profiles using both full annuli and annuli excluding a $120^{\circ}$ pie slice toward the west. All surface brightness profiles were computed using the location of the cross in Figure 1 as the origin. Data from the ACIS-S and ACIS-I observations were co-added and the normalized blank sky background images were used for background subtraction. To determine the appropriate weighting for the instrument and exposure maps, we extracted a spectrum from the central $500 \mathrm{kpc}$ of RCS $2318+0034$ and fit the spectrum to a single temperature apec model with the absorption fixed at the galactic value $\left(N_{\text {gal }}=4.04 \times 10^{20} \mathrm{~cm}^{-2}\right)$ and obtained kT=7.5 (6.4-8.6) keV, an abundance of $Z=0.12(<0.33)$ (based on the abundance table of Grevesse \& Sauval 1998) and an emission-measure of $\varepsilon=(3.21 \pm 0.16) \times 10^{-4} \mathrm{~cm}^{-5}$. All error bars are given at the $1 \sigma$ confidence level. Instrument and exposure maps weighted by the thermal emission from a $7.5 \mathrm{keV}$ plasma were then generated for all 5 CCDs used to calculate the surface brightness profiles. Figure 3 shows the resulting background-subtracted and exposure-corrected $0.5-6.0 \mathrm{keV}$ surface brightness profile excluding the emission from a $120^{\circ}$ pie slice toward the west. We refer to this as our standard surface brightness profile.

The X-ray surface brightness profile of clusters is commonly fit with a $\beta$ model, given by:

$$
\Sigma(r)=\Sigma_{0}\left(1+\left(\frac{r}{r_{c}}\right)^{2}\right)^{-3 \beta+1 / 2}
$$

The results of fitting a $\beta$ model to the background-subtracted and exposure-corrected surface brightness profiles using full annuli are shown in Table 1. This Table shows that as larger radii are included in the fitting process, larger values of $\beta$ and core radii, $r_{c}$, are obtained. The results of fitting the surface

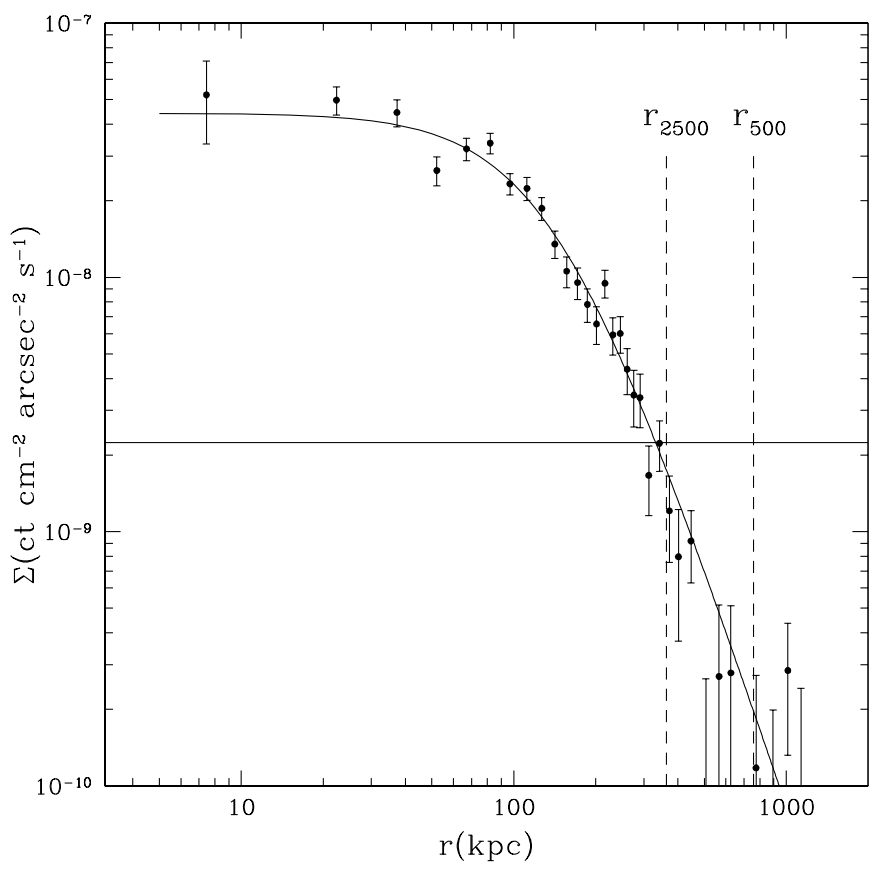

FIG. 3.- Background-subtracted and exposure-corrected 0.3-6.0 keV surface brightness profile of RCS $2318+0034$ excluding the emission from a $120^{\circ}$ pie slice toward the west along with our standard $\beta$ model (solid line). also shown are the ACIS-I background (horizontal solid line) and $r_{2500}$ and $r_{500}$ (vertical dashed lines).

brightness profiles that exclude the emission from a $120^{\circ}$ pie slice are shown in Table 2. This table shows that smaller values of $\beta$ and $r_{c}$ are obtained when the emission from the merging system toward the west is excluded, however, there is still a trend of increasing $\beta$ and $r_{c}$ when larger regions are included in the fitting process. The main difficulty in fitting the surface brightness profile is that the X-ray surface brightness of RCS $2318+0034$ drops below the I3 background around $350 \mathrm{kpc}$, which is only about 2 core radii from the center of the cluster (see Figure 3). It is therefore difficult to obtain a tight constraint on the asymptotic slope of the X-ray surface brightness.

To explore the systematic uncertainties in the derived parameters of the $\beta$ model, we generated a surface brightness profile excluding the emission from a $120^{\circ}$ pie slice, using an exposure map weighted by the thermal emission from a $4 \mathrm{keV}$ thermal plasma, since the gas temperature in clusters typically decreases by a factor of 2 at large radii (Vikhlinin et al. 2006). Table 2 shows that the derived values for the $\beta$ model are consistent with the values obtained using an exposure map weighted by the thermal emission from a $7.5 \mathrm{keV}$ plasma. We also varied the background level by $\pm 5 \%$ to determine the sensitivity of the derived parameters on the estimated background. This exercise shows that if the surface brightness profile is only fit within the central $350 \mathrm{kpc}$, then the results are fairly insensitive to the adopted background. However, if the emission from larger radii is included in the analysis, the results are quite sensitive to the exact background level used in the analysis.

\section{SPECTRAL ANALYSIS}

To further investigate the on-going merger in the center of RCS 2318+0034, we extracted spectra from the two cores and the region between the two cores. The results of fitting a single temperature apec model with the hydrogen column density fixed at the galactic value are shown in Table 3. While the uncertainties on the gas temperatures are fairly large, due to the 
TABLE 1

Surface Brightness Profile - Full ANNuli

\begin{tabular}{lccc}
\hline $\begin{array}{l}\text { Fitted Region } \\
(\mathrm{kpc})\end{array}$ & $\beta$ & $\begin{array}{c}\mathrm{r}_{\mathrm{c}} \\
(\mathrm{kpc})\end{array}$ & $\begin{array}{c}\Sigma_{0} \\
\left(\mathrm{ct} \mathrm{cm}^{-2} \mathrm{arcsec}^{-2} \mathrm{sec}^{-1}\right)\end{array}$ \\
\hline \hline $0-250$ & $0.64 \pm .11$ & $136 \pm 28$ & $(4.53 \pm 0.30) \times 10^{-8}$ \\
$0-350$ & $0.74 \pm .09$ & $159 \pm 23$ & $(4.42 \pm 0.26) \times 10^{-8}$ \\
$0-450$ & $0.85 \pm .09$ & $183 \pm 21$ & $(4.29 \pm 0.23) \times 10^{-8}$ \\
\hline
\end{tabular}

Notes: Results of fitting a $\beta$ model within different radii (Fitted Region) to the surface brightness profile extracted from within full annuli. The nominal background rates and instrument maps weighted by the emission from a $7.9 \mathrm{keV}$ thermal plasma were used in the analysis.

TABLE 2

Surface Brightness Profile - ExCluding A $120^{\circ}$ Pie Slice

\begin{tabular}{lccc}
\hline $\begin{array}{l}\text { Fitted Region } \\
(\mathrm{kpc})\end{array}$ & $\beta$ & $\begin{array}{c}r_{c} \\
(\mathrm{kpc})\end{array}$ & $\begin{array}{c}\Sigma_{0} \\
\left(\mathrm{ct} \mathrm{cm}^{-2} \mathrm{arcsec}^{-2} \mathrm{sec}^{-1}\right)\end{array}$ \\
\hline \hline $0-250$ & $0.58 \pm .09$ & $112 \pm 27$ & $(4.63 \pm 0.41) \times 10^{-8}$ \\
$0-350^{\dagger}$ & $0.70 \pm .09$ & $142 \pm 23$ & $(4.42 \pm 0.33) \times 10^{-8}$ \\
$0-450$ & $0.75 \pm .08$ & $152 \pm 21$ & $(4.36 \pm 0.23) \times 10^{-8}$ \\
\hline $0-250^{a}$ & $0.58 \pm .09$ & $112 \pm 26$ & $(5.39 \pm 0.47) \times 10^{-8}$ \\
$0-350^{a}$ & $0.70 \pm .09$ & $141 \pm 23$ & $(5.07 \pm 0.38) \times 10^{-8}$ \\
$0-450^{a}$ & $0.75 \pm .08$ & $152 \pm 21$ & $(4.99 \pm 0.36) \times 10^{-8}$ \\
\hline $0-250^{b}$ & $0.59 \pm .09$ & $114 \pm 27$ & $(4.60 \pm 0.40) \times 10^{-8}$ \\
$0-350^{b}$ & $0.73 \pm .10$ & $146 \pm 25$ & $(4.39 \pm 0.33) \times 10^{-8}$ \\
$0-450^{b}$ & $0.80 \pm .09$ & $161 \pm 23$ & $(4.30 \pm 0.31) \times 10^{-8}$ \\
\hline $0-250^{c}$ & $0.57 \pm .09$ & $110 \pm 26$ & $(4.65 \pm 0.41) \times 10^{-8}$ \\
$0-350^{c}$ & $0.68 \pm .08$ & $137 \pm 22$ & $(4.46 \pm 0.34) \times 10^{-8}$ \\
$0-450^{c}$ & $0.71 \pm .07$ & $144 \pm 19$ & $(4.41 \pm 0.32) \times 10^{-8}$ \\
\hline
\end{tabular}

Notes: Results of fitting a $\beta$ model within different radii (Fitted Region) to surface brightness profiles extracted from annuli that exclude the emission from a $120^{\circ}$ pie slice toward the west. The top 3 rows give the results using instrument maps weighted by the emission from a $7.5 \mathrm{keV}$ thermal plasma. ${ }^{a}$ Results using instrument maps weighted by the emission from a $3 \mathrm{keV}$ thermal plasma. ${ }^{b}$ Results obtained by increasing the background rates by $5 \% .{ }^{c}$ Results obtained by decreasing the background rates by $5 \%$. ${ }^{\dagger}$ Our standard $\beta$ model.

TABLE 3

Spectral Analysis of Selected Regions

\begin{tabular}{lc}
\hline Region & $\begin{array}{c}\mathrm{kT} \\
(\mathrm{keV})\end{array}$ \\
\hline \hline Western Core & $3.24(2.70-4.19)$ \\
Shock & $11.5(7.27-21.6)$ \\
Eastern Core & $7.18(4.36-14.7)$ \\
\hline
\end{tabular}

Notes: Best-fit temperatures and $1 \sigma$ uncertainties.

limited photon statistics, we find that the western core is cooler than the eastern core, as inferred from the hardness ratio map. There is also a region of hotter gas between the two cores. This is consistent with a merger scenario in which the hotter gas was produced by a merger shock. The large uncertainties in the temperature prevent a more detailed analysis of the merger dynamics.

A temperature profile was generated by extracting the emission from within 3 concentric annuli with approximately 1,000 net counts per spectrum. The spectra were fit to an absorbed single temperature apec model with the hydrogen column den- sity fixed at the galactic value. The resulting temperature profile shows some evidence for a radial decrease in the gas temperature (see Figure 4). Also shown in Figure 4 is the broken powerlaw temperature profile used to estimate the total gravitating mass in RCS 2318+0034 and the resulting values for $r_{2500}$ and $r_{500}$ (see $\S 6$ ). In addition, we extracted a spectrum from the innermost region excluding the emission within a $120^{\circ}$ pie slice toward the west (which excises the emission from the merging subcluster), but the resulting gas temperature is consistent with the inner temperature shown in Figure. 4. Since the emission from RCS $2318+0034$ drops below the X-ray 


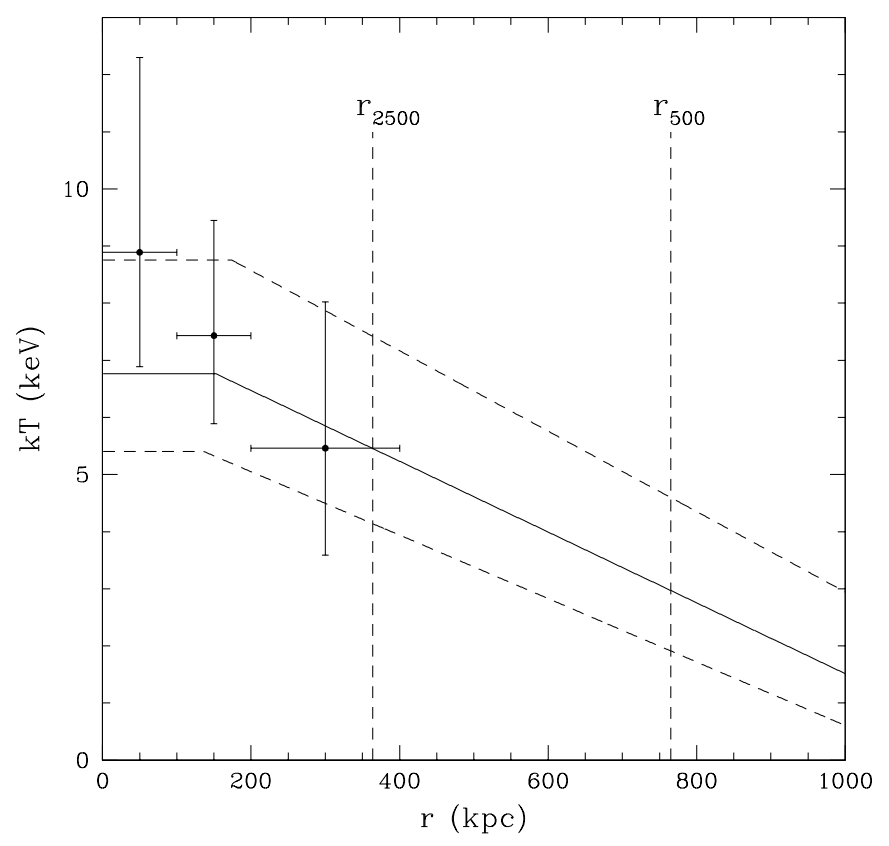

FIG. 4. - Temperature Profile of RCS 2318+0034 including the $1 \sigma$ error bars. The solid line shows the broken power-law temperature profile adopted from Vikhlinin et al. (2005) normalized by $T_{\text {spec }}$. The dashed lines show the broken broken power-law temperature profile normalized by the $1 \sigma$ limits on $T_{\text {spec }}$. The vertical dashed lines show $r_{2500}$ and $r_{500}$.

background at approximately $350 \mathrm{kpc}$, it is difficult to constrain the gas temperature at larger radii.

\section{GAS MASS, TOTAL MASS AND GAS MASS FRACTION}

Deprojecting the $\beta$ model surface brightness profile, assuming a temperature-independent emission-measure, produces a gas density profile of:

$$
\rho(r)=\rho_{0}\left(1+\left(\frac{r}{r_{c}}\right)^{2}\right)^{-3 \beta / 2}
$$

where $\rho_{0}$ is the central gas density. Thermal plasma models in $\mathrm{XSPEC}$ are normalized by the emission-measure, defined as:

$$
\varepsilon=\frac{10^{-14}}{4 \pi\left[D_{A}(1+z)\right]^{2}} \int n_{e} n_{H} d V
$$

where $D_{A}$ is the angular distance to the source, $n_{e}$ is the electron number density and $n_{H}$ is the hydrogen number density. The central gas density for a given $\beta$ model is determined by inserting the density profile given by eq. (2) into eq. (3), integrating over a cylindrical volume with a radius of $500 \mathrm{kpc}$ and equating the result with the observed emission-measure within this region. Using our standard $\beta$ model $\left(\beta=0.70\right.$ and $\left.r_{c}=142 \mathrm{kpc}\right)$ and the observed emission measure within $500 \mathrm{kpc}$, we obtain $n_{e}(0)=(8.04 \pm 0.18) \times 10^{-3} \mathrm{~cm}^{-3}$. The statistical uncertainty in the emission-measure produces an uncertainty of only $2.3 \%$ in the central gas density, since the density scales as the square root of the emission-measure. If we re-fit the spectrum extracted from the central $500 \mathrm{kpc}$ with the temperature fixed at 4 or $10 \mathrm{keV}$, we obtain best-fit emission-measures of $(3.47 \pm 0.22) \times 10^{-4} \mathrm{~cm}^{-5}$ or $(3.17 \pm 0.15) \times 10^{-4} \mathrm{~cm}^{-5}$, respectively. Thus, any variations in the gas temperature within this region would probably not generate more than an additional systematic uncertainty of $2.1 \%$ in the central gas density.

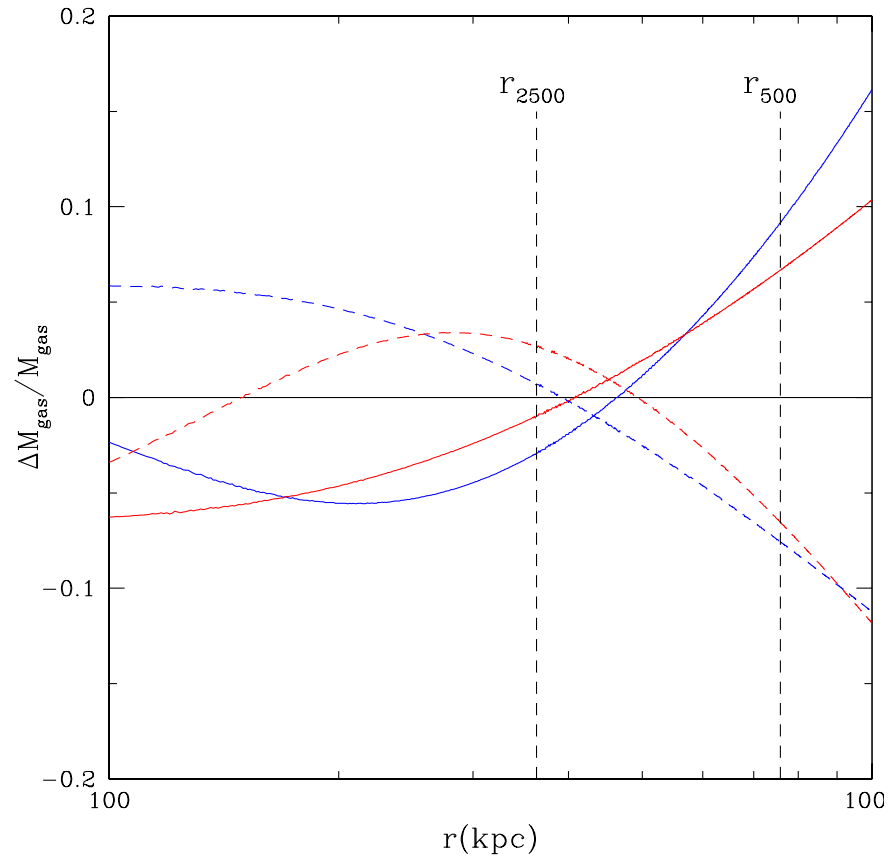

FIG. 5.- Ratio of the cumulative gas mass mass for several different $\beta$ models to the cumulative gas mass derived from our standard $\beta$ model ( $\beta=0.70$ and $\left.r_{c}=142 \mathrm{kpc}\right)$. The lines correspond to: solid red line $(\beta=0.64$ and $\left.r_{c}=136 \mathrm{kpc}\right)$, dashed red line $\left(\beta=0.85\right.$ and $\left.r_{c}=183 \mathrm{kpc}\right)$, sold blue line $\left(\beta=0.58\right.$ and $\left.r_{c}=112 \mathrm{kpc}\right)$ and dashed blue line $\left(\beta=0.75\right.$ and $\left.r_{c}=152 \mathrm{kpc}\right)$. The vertical dashed lines show $r_{2500}$ and $r_{500}$.

The dominant uncertainty in the gas mass in RCS 2318+0034 is not due to uncertainties in the central gas density, but is primarily due to the variations in the $\beta$ model parameters derived from fitting the $\mathrm{X}$-ray surface brightness over different regions (see the range in values of $\beta$ and $r_{c}$ in Tables 1 and 2). Figure 5 shows the cumulative gas mass derived from several different $\beta$ models relative to the cumulative gas mass derived from our standard $\beta$ model. The $\beta$ models used for this comparison are given in the figure caption and are chosen to sample the full range of possible gas masses. Figure 5 shows that the range of acceptable $\beta$ models produces an uncertainty in the gas masses at $r_{2500}$ and $r_{500}$ of about 5\% and 7-10\%, respectively. We thus assign an average combined uncertainty in the gas mass at these radii of $10 \%$.

The total gravitating mass of a spherically symmetric system in hydrostatic equilibrium is given by:

$$
M_{t o t}(<r)=\frac{-k T r}{\mu m_{p} G}\left(\frac{d \ln \rho}{d \ln r}+\frac{d \ln T}{d \ln r}\right)
$$

where $k$ is Boltzman's constant, $\mu=0.6$ is the mean mass per particle, $m_{p}$ is the proton mass and $G$ is the gravitational constant. The primary uncertainty in computing the gravitating mass in RCS $2318+0034$ is the lack of a detailed knowledge of the gas temperature profile. We therefore compute the gravitating mass assuming two temperature profiles: 1) an isothermal profile and 2) the broken power-law temperature profile in Vikhlinin et al. (2005). We use our standard $\beta$ model for the gas density distribution in both cases.

Extracting a spectrum in an annulus from $70 \mathrm{kpc}$ to $1 \mathrm{Mpc}$ in RCS 2318+0034 and fitting the spectrum to an absorbed apec model yields a temperature of $T_{\text {spec }}=6.32(5.05-8.18) \mathrm{keV}(1 \sigma$ uncertainty). The resulting gas mass, total gravitating mass and gas mass fraction at $r_{2500}$ and $r_{500}$ assuming the gas is isothermal are shown in Table 4 . The values for $r_{2500}$ and $r_{500}$ 
TABLE 4

RESUlts ASSUMING THE GAS IS ISOTHERMAL

\begin{tabular}{lcccccccc}
\hline $\begin{array}{l}\mathrm{T}_{\text {spec }} \\
(\mathrm{keV})\end{array}$ & $\begin{array}{c}\mathrm{r}_{2500} \\
(\mathrm{Mpc})\end{array}$ & $\begin{array}{c}\mathrm{r}_{500} \\
(\mathrm{Mpc})\end{array}$ & $\begin{array}{c}\mathrm{M}_{\text {gas }}\left(\mathrm{r}_{2500}\right) \\
\left(10^{13} \mathrm{M}_{\odot}\right)\end{array}$ & $\begin{array}{c}\mathrm{M}_{\text {gas }}\left(\mathrm{r}_{500}\right) \\
\left(10^{13} \mathrm{M}_{\odot}\right)\end{array}$ & $\begin{array}{c}\mathrm{M}_{\text {tot }}\left(\mathrm{r}_{2500}\right) \\
\left(10^{13} \mathrm{M}_{\odot}\right)\end{array}$ & $\begin{array}{c}\mathrm{M}_{\text {tot }}\left(\mathrm{r}_{500}\right) \\
\left(10^{13} \mathrm{M}_{\odot}\right)\end{array}$ & $\mathrm{f}_{\text {gas }}\left(\mathrm{r}_{2500}\right)$ & $\mathrm{f}_{\text {gas }}\left(\mathrm{r}_{500}\right)$ \\
\hline \hline 5.05 & 0.308 & 0.745 & 0.796 & 2.82 & 9.96 & 28.2 & 0.080 & 0.10 \\
6.32 & 0.352 & 0.837 & 0.992 & 3.25 & 14.8 & 39.9 & 0.067 & 0.081 \\
8.18 & 0.408 & 0.955 & 1.25 & 3.81 & 23.1 & 59.3 & 0.054 & 0.064 \\
\hline
\end{tabular}

Notes: The gas mass $\left(M_{\text {gas }}\right)$, total gravitating mass $\left(M_{t o t}\right)$ and gas mass fraction $\left(f_{\text {gas }}\right)$ within $r_{2500}$ and $r_{500}$ derived from our standard $\beta$ model and the assumption that the gas is isothermal. Masses are shown for the best-fit emission-weighted temperature between $70 \mathrm{kpc}$ and $1 \mathrm{Mpc}\left(T_{\text {spec }}\right)$ and the $1 \sigma$ limits on $T_{\text {spec }}$.

TABLE 5

Results Using a Broken Power-Law Temperate Profile

\begin{tabular}{lcccccccc}
\hline $\begin{array}{l}\mathrm{T}_{\text {spec }} \\
(\mathrm{keV})\end{array}$ & $\begin{array}{c}\mathrm{r}_{2500} \\
(\mathrm{Mpc})\end{array}$ & $\begin{array}{c}\mathrm{r}_{500} \\
(\mathrm{Mpc})\end{array}$ & $\begin{array}{c}\mathrm{M}_{\text {gas }}\left(\mathrm{r}_{2500}\right) \\
\left(10^{13} \mathrm{M}_{\odot}\right)\end{array}$ & $\begin{array}{c}\mathrm{M}_{\text {gas }}\left(\mathrm{r}_{500}\right) \\
\left(10^{13} \mathrm{M}_{\odot}\right)\end{array}$ & $\begin{array}{c}\mathrm{M}_{\text {tot }}\left(\mathrm{r}_{2500}\right) \\
\left(10^{13} \mathrm{M}_{\odot}\right)\end{array}$ & $\begin{array}{c}\mathrm{M}_{\text {tot }}\left(\mathrm{r}_{500}\right) \\
\left(10^{13} \mathrm{M}_{\odot}\right)\end{array}$ & $\mathrm{f}_{\text {gas }}\left(\mathrm{r}_{2500}\right)$ & $\mathrm{f}_{\text {gas }}\left(\mathrm{r}_{500}\right)$ \\
\hline \hline 5.05 & 0.320 & 0.682 & 0.853 & 2.53 & 11.2 & 21.6 & 0.076 & 0.12 \\
6.32 & 0.364 & 0.765 & 1.05 & 2.93 & 16.4 & 30.4 & 0.064 & 0.096 \\
8.18 & 0.420 & 0.872 & 1.30 & 3.43 & 25.1 & 45.1 & 0.052 & 0.076 \\
\hline
\end{tabular}

Notes: The gas mass $\left(M_{\text {gas }}\right)$, total gravitating mass $\left(M_{t o t}\right)$ and gas mass fraction $\left(f_{\text {gas }}\right)$ within $r_{2500}$ and $r_{500}$ derived from our standard $\beta$ model along with the temperature profile in Vikhlinin et al. (2005). Masses are shown for the the best-fit emission-weighted temperature $\left(T_{\text {spec }}\right)$ and the $1 \sigma$ limits on $T_{\text {spec }}$.

are calculated from the total gravitating mass profile. Since the resulting values for $r_{500}$ in Table 4 are slightly less than $1 \mathrm{Mpc}$, we also extracted spectra from several annuli with inner radii of $70 \mathrm{kpc}$ and outer radii of 700,800 and $900 \mathrm{kpc}$. Due to the limited photon statistics in the Chandra observation, the best-fit temperatures are all consistent within the uncertainties. Table 4 shows that assuming an isothermal $\beta$ model for RCS $2318+0034$ produces gas mass fractions of $0.07 \pm .02$ and $0.08 \pm .02$ at $r_{2500}$ and $r_{500}$, respectively. The error bars take into account the $10 \%$ uncertainty in the gas mass noted above and the uncertainty in $T_{\text {spec }}$. The gas mass fraction is essentially independent of radius at large radii since, for an isothermal $\beta=0.7$ model, $\rho_{\text {gas }} \sim r^{-2.1}$ and $\rho_{\text {tot }} \sim r^{-2}$.

Based on the analysis of Chandra observations of a sample of 13 rich clusters of galaxies, Vikhlinin et al. (2005) found that the temperature profile of these clusters could be fit with a broken power-law model, given by:

$$
T /\langle T\rangle= \begin{cases}1.07, & 0.035<r / r_{180}<0.125, \\ 1.22-1.2 r / r_{180}, & 0.125<r / r_{180}<0.6 .\end{cases}
$$

where $\langle T\rangle$ is the emission-weighted temperature excluding the central $70 \mathrm{kpc}$ (essentially $T_{\text {spec }}$ ). For clusters, $r_{500} \sim 0.6 r_{180}$. With this substitution, the temperature profile in eq. (5) is very similar to that derived for a sample of groups by Sun et al. (2009).

Gas masses, total gravitating masses and gas mass fractions within $r_{2500}$ and $r_{500}$ are shown in Table 5 using our standard $\beta$ model and the broken power-law temperature profile in eq. (5) normalized by $T_{\text {spec }}$. Since eq. (5) depends on $r_{500}$, we iterated in $r_{500}$ until a self-consistent solution was found. The selfconsistent temperature profiles are shown in Figure 4. Table 5 shows that assuming a broken power-law temperature profile for RCS $2318+0034$ produces gas mass fractions of $0.06 \pm .02$ and $0.10 \pm .02$ at $r_{2500}$ and $r_{500}$, respectively. The error bars take into account the $10 \%$ uncertainty in the gas mass and the uncertainty in $T_{\text {spec }}$. As opposed to the isothermal model, the gas mass fraction increases with radius due to the declining gas temperature.

While RCS $2318+0034$ is presently undergoing a merger, Nagai et al. (2007) have shown by performing mock Chandra observations of a sample of simulated clusters that X-ray derived gas masses in both relaxed and unrelaxed clusters are accurate to within $6 \%$. Including this additional uncertainty in our derived gas mass would not a have a significant affect on our estimate of the gas mass fraction. Nagai et al. (2007) also found that X-ray derived gravitating masses are biased low by about $5-20 \%$ with no trend in cluster mass or redshift. This suggests that all X-ray derived gas mass fraction estimates are biased high by this amount.

\section{COMPARISON WITH PREVIOUS ANALYSIS}

Our estimated gas mass fractions using the combined $200 \mathrm{ksec}$ of Chandra data are approximately $50 \%$ greater at $r_{2500}$ and significantly greater at $r_{500}$ compared with the values derived from the earlier $50 \mathrm{ksec}$ observation (Hicks et al. 2008). Comparing the derived parameters for RCS $2318+0034$ in Hicks et al. with our results, we find that both studies produced similar values for the gas temperature $\left(T_{\text {spec }}=5.05-\right.$ $8.18 \mathrm{keV}$ compared to $T_{\text {spec }}=5.20-7.40 \mathrm{keV}$ ) and core radius ( $r_{c}=142 \pm 23 \mathrm{kpc}$ compared to $r_{c}=171 \pm 6 \mathrm{kpc}$ ) for the surface brightness profile. However, we obtain a value for the outer slope of the surface brightness profile corresponding to $\beta=0.70 \pm .09$ compared to $\beta=0.86 \pm .03$ in Hicks et al. This difference is primarily due to the poorer photon statistics in the initial $50 \mathrm{ksec}$ Chandra observation. Larger values of $\beta$ will over estimate the total gravitating mass and underestimate the gas mass and gas mass fraction. Jones \& Forman (1999) found an average value of $\beta=0.6$ when fitting the surface brightness 
profile of a large sample of rich clusters observed with

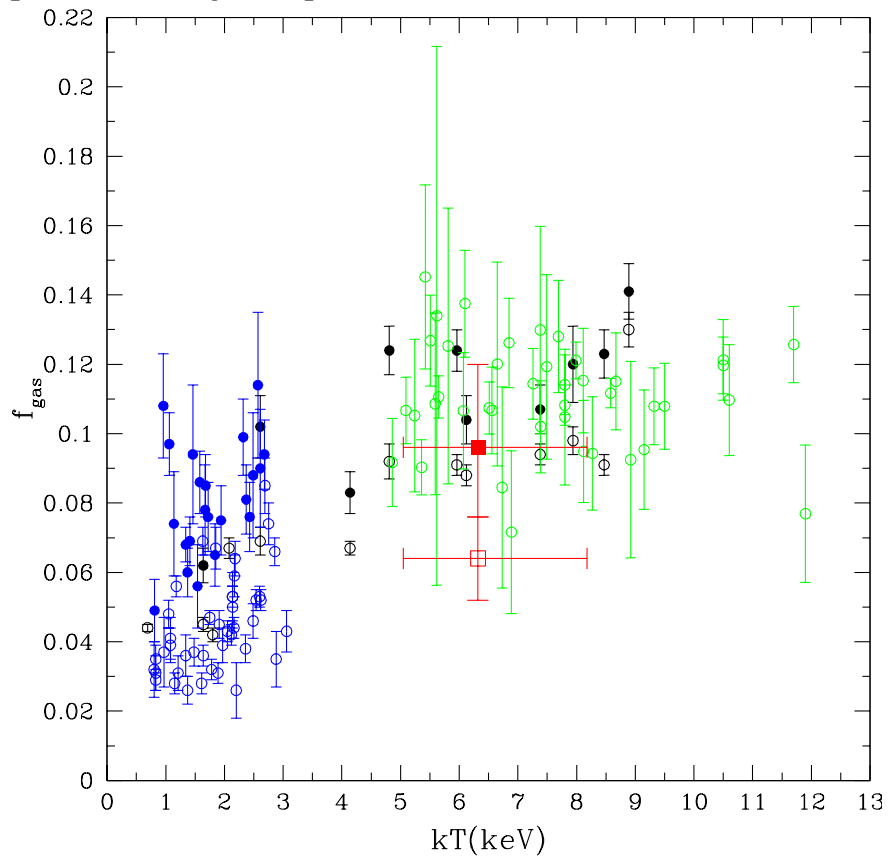

FIG. 6.- Gas mass fractions from Sun et al. (2009) (blue), Vikhlinin et al. (2006) (black), Allen et al. (2009) (green) and RCS 2318+0034 (red). Open symbols give $f_{\text {gas }}$ within $r_{2500}$ and solid symbols give $f_{\text {gas }}$ within $r_{500}$.

the Einstein Observatory. More recently, in a study of 31 clusters culled from the REXCESS sample, which includes both relaxed and unrelaxed clusters, Croston et al. (2008) found an average power-law slope in the surface brightness profile between $0.3 r_{500}$ and $0.8 r_{500}$ among $6 \mathrm{keV}$ clusters corresponding to $\beta=0.7$.

At $r_{2500}$, we obtain $M_{\text {gas }}\left(r_{2500}\right)=9.9_{-2.0}^{+2.6} \times 10^{12} \mathrm{M}_{\odot}$ and $M_{\text {tot }}\left(r_{2500}\right)=1.5_{-0.5}^{+0.8} \times 10^{14} \mathrm{M}_{\odot}$ assuming the gas is isothermal. Hicks et al. (2008) derived $M_{\text {gas }}\left(r_{2500}\right)=(9.7 \pm 0.6) \times$ $10^{12} \mathrm{M}_{\odot}$ and $M_{t o t}\left(r_{2500}\right)=(2.43 \pm 0.28) \times 10^{14} \mathrm{M}_{\odot}$ assuming the gas is isothermal. However, the gas and total masses listed for RCS $2318+0034$ in Table 7 of Hicks et al. are inconsistent with the parameters given in Tables 3 and 4 . The corrected values are $M_{\text {gas }}\left(r_{2500}\right)=1.60 \times 10^{13} \mathrm{M}_{\odot}$ and $M_{\text {tot }}\left(r_{2500}\right)=2.80 \times$ $10^{14} \mathrm{M}_{\odot}$ (Hicks 2011, private communication). While there are some differences between our results due to differences in $r_{2500}$ and $\beta$, our gas mass fraction at $r_{2500}$ is consistent with the corrected Hicks (2011, private communication) result. We find that assuming a broken power-law temperature profile increases both $M_{\text {gas }}\left(r_{2500}\right)$ and $M_{\text {tot }}\left(r_{2500}\right)$ by $10 \%$ relative to the values derived by assuming the gas is isothermal. Thus, both temperature profiles produce the same value for the gas mass fraction of $f_{\text {gas }}\left(r_{2500}\right)=0.06 \pm 0.02$.

At $r_{500}$, we obtain $M_{\text {gas }}\left(r_{500}\right)=3.2_{-0.7}^{+1.0} \times 10^{13} \mathrm{M}_{\odot}$, $M_{\text {tot }}\left(r_{500}\right)=4.0_{-1.2}^{+1.9} \times 10^{14} \mathrm{M}_{\odot}$ and $f_{\text {gas }}\left(r_{500}=0.06 \pm 0.02\right.$ assuming the gas is isothermal. Hicks et al. (2008) derived $M_{\text {gas }}\left(r_{500}\right)=(1.99 \pm 0.28) \times 10^{13} \mathrm{M}_{\odot}$ and $M_{\text {tot }}\left(r_{500}\right)=$ $(12.9 \pm 2.0) \times 10^{14} \mathrm{M}_{\odot}$. However, the corrected values are $M_{\text {gas }}\left(r_{500}\right)=3.64 \times 10^{13} \mathrm{M}_{\odot}$ and $M_{\text {tot }}\left(r_{500}\right)=6.61 \times 10^{14} \mathrm{M}_{\odot}$ (Hicks 2011, private communication). Assuming a broken power-law temperature profile reduces $M_{g a s}\left(r_{500}\right)$ by $10 \%$ (due to the smaller value of $\left.r_{500}\right)$ and $M_{t o t}\left(r_{500}\right)$ by $25 \%$ (primarily due to the declining gas temperature). The assumption of a broken power-law temperature profile thus produces a larger gas mass fraction of $f_{\text {gas }}\left(r_{500}\right)=0.10 \pm 0.02$.

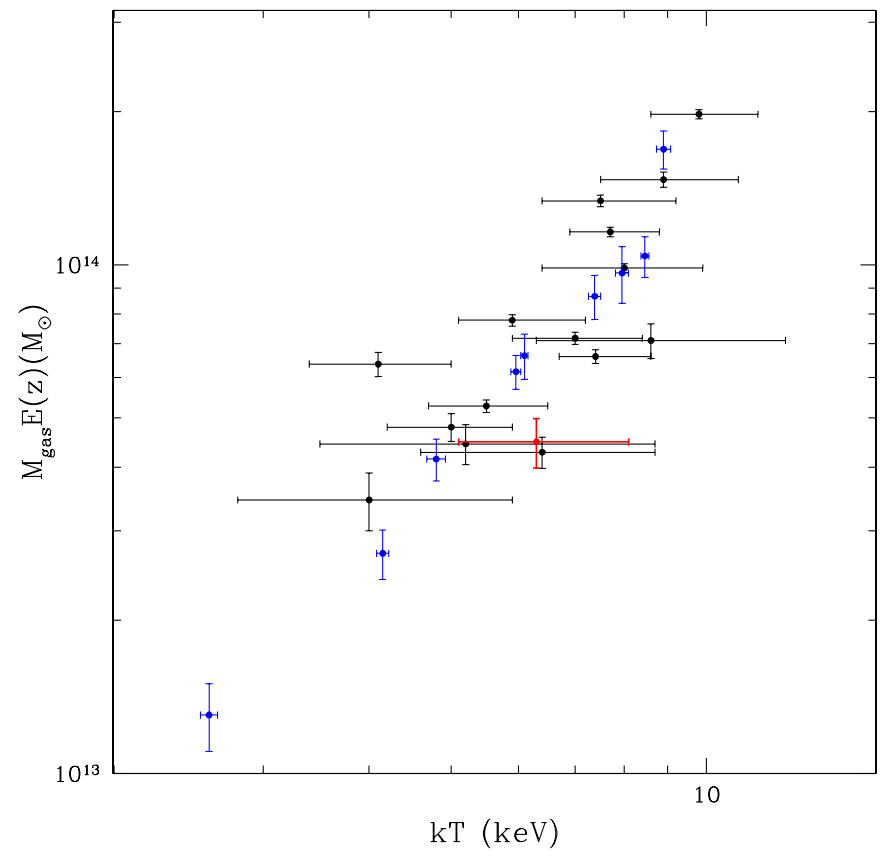

FIG. 7.- Scaled gas mass vs. spectroscopic temperature for the X-ray selected clusters in Vikhlinin et al. (2006) (blue data points), the SZ-selected clusters in Andersson et al. (2010) (black data points) and the opticallyselected cluster RCS 2318+0034 (red data point).

\section{DISCUSSION AND SUMMARY}

In general, the gas mass fraction in groups and clusters depends on the radius at which the gas mass fraction is computed and the virial mass of the cluster. At small radii, the gas mass fraction also depends on whether the cluster has a cool-core. The central gas density in cool-core clusters is more than an order of magnitude greater than the central density in non-cool core systems. Since $r_{2500}$ is only $\sim 200 \mathrm{kpc}$ in groups, the presence of a cool-core can have a significant impact on the gas mass fraction at $r_{2500}$ in groups. Sun et al. (2206) shows that there is indeed a strong anti-correlation between the gas mass fraction in groups at $r_{2500}$ and the central gas entropy. For hotter systems (larger values of $r_{2500}$ ), the presence of a cool-core has less of an impact on the gas mass fraction at $r_{2500}$.

Reports of the average gas mass fraction within $r_{2500}$ vary from $4.5 \%$ in groups (Sun et al. 2009), up to $7.8 \%$ (Vikhlinin et al. 2006) and $11.0 \%$ (Allen et al. 2008) in clusters. The reported gas mass fraction within $r_{500}$ varies from $8.1 \%$ in groups (Sun et al. 2009), up to $13.0 \%$ in clusters (Vikhlinin et al. 2006, Ettori et al. 2010, Pratt et al. 2010, Juett et al. 2010). These results are illustrated in Figure 6 and show that the differences are mainly due to the range in gas temperatures, or cluster masses, sampled in these different studies. The average gas temperature of the clusters in the Vikhlinin et al. sample is $4.5 \mathrm{keV}$ compare to $7.7 \mathrm{keV}$ in the sample of clusters analyzed by Allen et al., which contains a substantial fraction of clusters culled from the Massive Cluster Survey (MACS; Ebeling et al. 2001).

Our estimate for the gas mass fraction within $r_{500}$ in the optically selected cluster, RCS2318+0034, is fully consistent with the results of X-ray selected clusters (see Figure 6). This result is also illustrated in Figure 7, where we compare the scaled gas mass and spectroscopic temperature within $r_{500}$ for the Xray selected clusters in Vikhlinin et al. (2006), the SZ-selected 
clusters in Andersson et al. (2010) and the optically selected cluster, RCS 2318+0034. Croston et al. (2008) derived a

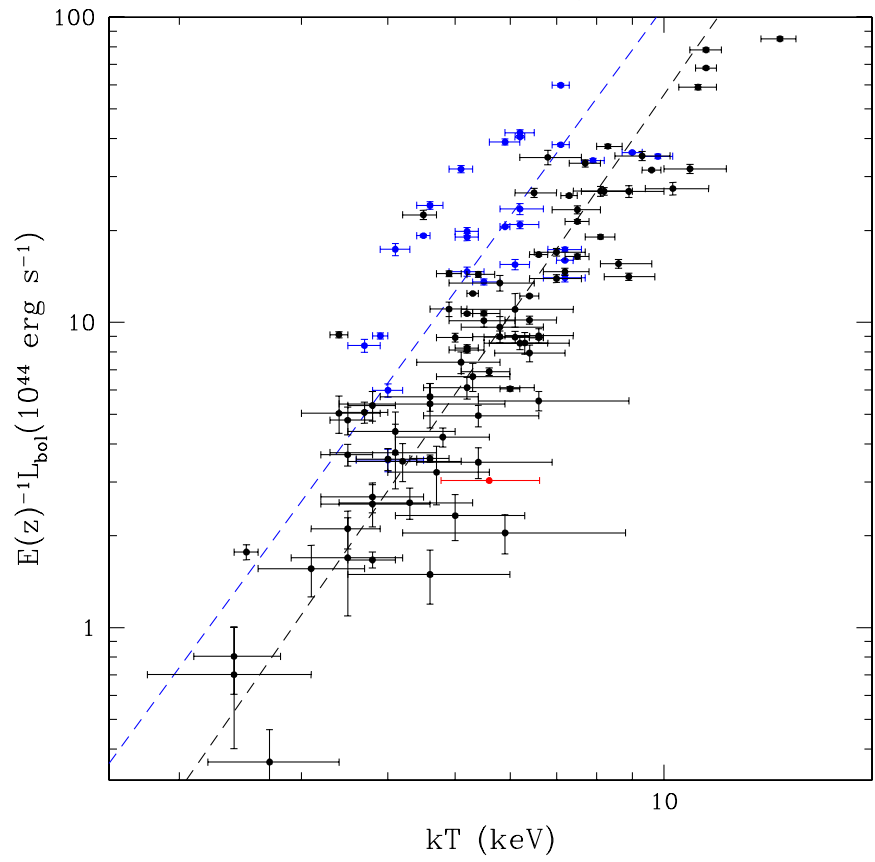

FIG. 8.- Bolometric X-ray luminosity vs. cluster temperature relation for the total emission within $r_{500}$. The RCS $2318+0034$ data point is shown in red. All other data points are from Maughan et al. (2011). Relaxed clusters are shown in blue and unrelaxed clusters are shown in black. Also shown are the best fit relations for relaxed clusters (dashed blue line) and unrelaxed clusters (dashed black line) derived in Maughan et al.

scaling relation between the gas mass and temperature based on an analysis of clusters culled from the REXCESS sample. The scaling relation in Croston et al. is based on the temperature between $0.15 r_{500}$ and $0.75 r_{500}$. Extracting a spectrum from within this region in RCS2318+0034 gives a temperature of $5.3 \pm 091 \mathrm{keV}$. Using the scaling relations in Croston et al. gives an estimated gas mass at $r_{500}$ of $(2.2-4.2) \times 10^{13} \mathrm{M}_{\odot}$, which is consistent with our estimate for the gas mass in RCS2318+0034 (see Table 4). In addition, the scaling relation between $f_{\text {gas }}\left(r_{500}\right)$ and $M_{\text {tot }}\left(r_{500}\right)$ derived by Vikhlinin et al. (2009) predicts $f_{\text {gas }}\left(r_{500}\right)=10.0 \%$, which is in very good agreement with our result. Within $r_{2500}$, we find a gas mass fraction of $6 \%$ for RCS $2318+0034$, which is significantly less than that found for most clusters (see Figure 6). Figures 1 and 2 show that RCS2318+0034 is undergoing a merger which may affect the estimate of the gas mass fraction at $r_{2500}$.

One of the most extensively studied cluster relations is that between the gas temperature and X-ray luminosity (e.g., Edge \& Stewart 1991, David et al. 1993; Fabian et al. 1994; Markevitch 1998, Arnaud \& Evrard 1999). Early studies found a great deal of scatter in the Lx-T relation (Edge \& Stewart 1991; David et al. 1993). Fabian et al. (1994) showed that most of this scatter was due to the inclusion of massive cooling flow clusters along with non cool-core clusters in the previous studies. The $\mathrm{X}$-ray luminosity of massive cooling flow clusters can be up to five times greater than the X-ray luminosity of non cool-core clusters for a given temperature. By removing the emission from cool cores, Markevitch (1998) found that the scatter in the Lx-T relation can be greatly reduced. Similar conclusions have also been obtained by more recent studies (e.g., Maughan 2007, Prat et al. 2009, Maughan et al. 2011). While most studies have shown that the Lx-T relation for clusters at a given redshift is not self-similar, there is significant evidence that the evolution of the Lx-T relation is nearly self-similar (e.g., Vikhlinin et al. 2002, Maughan 2006, Maughan et al. 2011).

Maughan et al. (2011) recently studied the Lx-T relation for a sample of 114 clusters observed by Chandra with redshifts of $0.1<z<1.3$. The Lx-T relations in Maughan et al. were derived from both the total emission within $r_{500}$ and the emission within $(0.15-1.0) r_{500}$. Due to the limited photon statistics in the Chandra observation of RCS2318+0034, the uncertainty in the gas temperature between $(0.15-1.0) r_{500}$ is too large for a useful comparison, so we only compare results using the total emission within $r_{500}$. Figure 8 shows the scaled Lx-T data for the relaxed and unrelaxed clusters in Maughan et al. (2011) along with the temperature and X-ray luminosity of RCS2318+0034. Figure 8 shows that the temperature and $\mathrm{X}$-ray luminosity of the $\mathrm{z}=0.78$ cluster, $\mathrm{RCS} 2318+0034$, is consistent with other unrelaxed clusters with similar redshifts given the observed scatter among clusters.

In summary, we find that the large scale properties of the optically selected cluster RCS2318+0034 are consistent with the large scale properties of X-ray selected clusters. At smaller radii, we find a lower gas mass fraction in RCS2318+0034 compared to most X-ray selected clusters, which may be due to the on-going merger.

We would like to than A. Hicks for her help with comparing our results and also A. Vikhlinin for useful comments. This work was supported in part by the NASA grant GO8-9122X.

\section{REFERENCES}

Allen, S., Schmidt, R., Ebeling, H., Fabian, A., van Speybroeck, L. 2004, MNRAS, 353, 457.

Allen, S., Rapetti, D., Schmidt, R., Ebeling, H., Morris, R. \& Fabian, A. 2008, MNRAS, 383, 879.

Andersson, K. et al. 2010 (astro-ph 1006.3068).

Arnaud, M. \& Evrard, A. 1999, MNRAS, 305, 631

Ascasibar, Y. \& Markevitch, M. 2006, ApJ, 650, 102.

Bialek, J., Evrard, A. E., Mohr, J. 2001, ApJ, 555, 597.

Bode, P., Ostriker, J. \& Vikhlinin, A. 2009, ApJ, 700, 989.

Croston, J., Pratt, G., Bohringer, H., Arnaud, M., Pointecouteau, E., Ponman, T., Sanderson, A., Temple, R., Bower, R. \& Donahue, M. 2008, A\&A, 487, 431.

Davé, R., Oppenheimer, B., Sivanandam \& S. 2008, MNRAS, 391110.

David, L., Arnaud, K., Forman, W. \& Jones, C. 1990, ApJ, 356, 32.

David, L., Slyz, A., Jones, C., Forman, W., Vrtilek, S. \& Arnaud, K. 1993, ApJ, $412,479$.

David, L. Jones, C. \& Forman, W. 1995, ApJ, 445, 578.

Ebeling, H., Edge, A. \& Henry, J. 2001, ApJ, 553, 668.

Edge, A. \& Stewart, G. 1991, MNRAS, 252, 414.
Ettori, S., Tozzi, P. \& Rosati, P. 2003, A\&A, 398, 879.

Ettori, S., Morandi, A., Tozzi, P., Balestra, I., Borgani, S., Rosati, P., Lovisari, L.\& Terenziani, F. 2009, A\&A, 501, 61.

Ettori, S., Gastaldello, F., Leccardi, A., Molendi, S., Rossetti, M., Buote, D. \& Meneghetti, M. 2010, A\&A, 524, 68.

Fabian, A., Crawford, C., Edge, A. \& Mushotzky, R. 2004, MNRAS, 267, 779.

Ferramacho, L. \& Blanchard, A. 2007, A\&A, 463, 423.

Finoguenov, A.; Jones, C., Böhringer, H. \& Ponman, T. 2002, ApJ, 578, 74.

Gladders, M. \& Yee, H. 2005, ApJS, 157, 1.

Gonzalez, A., Zaritsky, D., Zabludoff, A. 2007, ApJ, 666, 147.

Hicks, A., Ellingson, E., Bautz, M., Cain, B., Gilbank, D., Gladders, M., Hoekstra, H., Yee, H. \& Garmire, G. 2008, ApJ, 680, 1022.

Hicks, A., 2011, private communication.

Jones, C. \& Forman, W. 1999, ApJ., 511, 65

Juett, A., Davis, D. \& Mushotzky, R. 2010, ApJ, 709, 103.

Kravtsov, A., Andrey V., Nagai, D. \& Vikhlinin, A. 2005, 2005, ApJ, 625, 588.

Lloyd-Davies, E., Ponman, T. \& Cannon, D. 2000, MNRAS, 315, 689.

Markevitch, M. 1998, ApJ, 504, 27.

Maughan, B. 2007, ApJ, 668, 772. 
Maughan, B., Giles, P., Randall, S., Jones, C. \& Forman, W. 2011 (astro-ph 1108.1200)

Nagai D., Vikhlinin, A. \& Kravtsov, A. 2007, ApJ, 655, 98.

Neumann, D. \& Arnaud, M. 2001, A\&A, 373, 33.

Ponman, T., Cannon, D. \& Navarro, J. 1999, Nature, 397, 135.

Pratt, G., Croston, Arnaud, M. \& Bohringer, H. 2009, A\&A, 361, 378

Pratt, G., Arnaud, M., Piffaretti, R., Bohringer, H., Ponman, T., Croston, J., Voit, G., Borgani, S. \& Bower, R. 2010, A\&A, 511, 85.

Sun, M., Voit, G., Donahue, M., Jones, C., Forman, W. \& Vikhlinin, A. 2009, ApJ, 693, 1142.
Vikhlinin, A., van Speybroeck, L., Markevitch, M., Forman, W. \& Grego, L. 2002, ApJ, 578, 107.

Vikhlinin, A., Kravtsov, A., Forman, W., Jones, C., Markevitch, M., Murray, S. \& Van Speybroeck, L. 2006, ApJ, 640, 691.

Vikhlinin, A., Burenin, R., Ebeling, H., Forman, W, Hornstrup, A., Jones, C. Kravtsov, A., Murray, S., Nagai, D., Quintana, H. \& Voevodkin, A. 2009, ApJ, 692, 1033.

Voit, G. M., Kay, S. \& Bryan, G. 2005, MNRAS, 364, 909.

White, D. \& Fabian, A. 1995, MNRAS, 273, 72

White, S. Navarro, J., Evrard, A. E. \& Frenk, C. 1993, Nature, 366, 429. 